Article

\title{
Self-Metathesis of Methyl Oleate Using Ru-NHC Complexes: A Kinetic Study
}

\author{
Marc Renom Carrasco ${ }^{1,+}+\mathbb{D}$, Clémence Nikitine ${ }^{2,+}+\mathbb{C}$, Mohamed Hamou ${ }^{2,+}+\mathbb{D}$, \\ Claude de Bellefon ${ }^{2, *,+} \mathbb{1}$, Chloé Thieuleux ${ }^{1, *,+}$ (1) and Valérie Meille ${ }^{2, *, t, \neq}$ \\ 1 Laboratory of Chemistry, Catalysis, Polymers and Processes (C2P2), Institut de Chimie de Lyon, \\ Université de Lyon, UMR 5265 CNRS-UCB Lyon 1-CPE Lyon, CPE Lyon, 43 Bd du 11 Novembre 1918, \\ F-69616 Villeurbanne, France; mrenom5@alumno.uned.es \\ 2 Laboratoire de Génie des Procédés Catalytiques, Institut de Chimie de Lyon, Université de Lyon, \\ UMR 5285 CNRS-UCB Lyon 1-CPE Lyon, CPE Lyon, 43 Bd du 11 Novembre 1918, \\ F-69616 Villeurbanne, France; cni@lgpc.cpe.fr (C.N.); mha@lgpc.cpe.fr (M.H.) \\ * Correspondence: cdb@lgpc.cpe.fr (C.d.B.); thieuleux@cpe.fr (C.T.); \\ valerie.meille@ircelyon.univ-lyon1.fr (V.M.) \\ + These authors contributed equally to this work. \\ $\ddagger$ Current address: Université de Lyon, Université Claude Bernard Lyon 1, CNRS, IRCELYON, \\ F-69626 Villeurbanne, France.
}

Received: 31 March 2020; Accepted: 12 April 2020; Published: 17 April 2020

\begin{abstract}
A kinetic study concerning the self-metathesis of methyl oleate and methyl elaidate was performed, using a variety of NHC-ruthenium pre-catalysts, bearing either mesityl groups or di-isopropyl-phenyl groups on the NHC ligand and various trans ligands with respect to the NHC unit. We showed that the system can be satisfactorily described using one initiation constant per pre-catalyst and four propagation constants that, conversely, do not depend on the pre-catalyst. The difference of reactivity with oleate $(Z)$ and elaidate $(E)$ can be fully explained by the propagation parameters; the studied pre-catalysts initiate with the same rate starting from the $Z$ or the $E$ olefin. The ranking of the propagation parameters is driven by the thermodynamic equilibrium. The transformation rates of $Z$ and $E$ isomers is only driven by these propagation constants and nothing differentiates the initiation step.
\end{abstract}

Keywords: olefin metathesis; ruthenium; kinetics; initiation; methyl oleate

\section{Introduction}

Kinetic studies of self-metathesis (or homo-metathesis) reactions with Ru catalysts are mainly dedicated to the transformation of terminal olefins, producing ethylene and a symmetrical internal olefin [1-3]. The fate of ethylene in these literature precedents is not always described, and the concentration of dissolved ethylene in the liquid mixture may vary from an experiment to another. Very few studies concern the kinetic study of homo-metathesis of internal olefins, with no gaseous product formation. However, kinetic data would be helpful to better analyze the contributions of the ligands on the initiation and propagation steps, and thus to rationally propose ligand improvements. Here, the chosen reaction is the homo-metathesis of methyl 9-octadecenoate (AB) catalyzed by ruthenium complexes (Scheme 1). In its $Z$ configuration, $A B$ is named methyl oleate, and, in its $E$ configuration, methyl elaidate. The reaction produces 9-octadecene (AA) and dimethyl-9-octadecene-1,18-dioate (BB). The symmetrical olefin can be useful in the lube-oil range hydrocarbons or in candle waxes after hydrogenation, whereas the di-ester can find an outlet in the synthesis of polyesters, polyamides, and cosmetics [4-6]. 
2

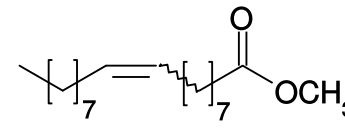

$A B$

methyl 9-octadecenoate

methyl oleate $(A B Z) /$ methyl elaidate $(A B E)$<smiles>CCCC=CCCC</smiles>

$\mathrm{AA}$

9-octadecene

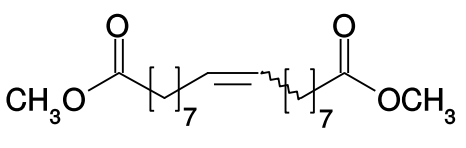

BB

dimethyl-9-octadecene-1,18-dioate

Scheme 1. Methyl 9-octadecenoate self-metathesis.

In the published works, the reaction was studied either with molecular catalysts [7-9] or with supported catalysts $[10,11]$. The objectives were to compare the activity, selectivity, and stability of the catalysts but scarcely to go into kinetic data interpretation. Some kinetic studies using methyl oleate (Z-methyl 9-octadecenoate) were nevertheless performed [12,13] but they did not concern $\mathrm{Ru}$ catalysts. The mechanism of the metathesis reaction has been extensively studied, involving several consecutive steps: an initiation (reverse reaction) that transforms part of the pre-catalysts into catalytic active species, a propagation step being the catalytic cycle itself, and a recombination step that restores the pre-catalytic species. Many publications are dedicated to the initiation kinetics of the Hoveyda-Grubbs pre-catalysts [1,14-18]. The steps are summarized in Scheme 2. For the sake of conciseness, the mechanism is presented for an internal olefin $\mathrm{R}=\mathrm{R}$ (the two Rs being equal or different and the olefin being $Z$ or $E$ ).

Based on literature precedents, we consider that: (i) the mechanism starts with a 16-electron Ru-NHC complex 1; and (ii) the energy of the bottom-bond reaction pathway, where the olefin is coordinated trans to the NHC ligand, is considerably lower than the energy of the side-bond pathway, where the olefin is coordinated cis to the NHC ligand $[16,19]$. The dissociative mechanism proposes that the ligand L' dissociation occurs first to give the 14-electron species, followed by the olefin coordination to regenerate a 16-electron species 4 . This mechanism is in general preferred compared to the associated one (leading to 3 ) in the case of sterically demanding olefins such as methyl 9-octadecenoate $[14,15]$. Then, to complete the initiation mechanism, a first metathesis reaction allows replacing the initial $\mathrm{R}^{\prime}$ ligand by one of the $\mathrm{R}$ alkylidene fragments from the substrate. The 14-electron 7 species, similar to 4 , but containing only the $\mathrm{R}$ alkylidene fragment can then enter into the propagation loop. In the present work, a global kinetic approach was followed to evaluate the kinetic constants allowing to describe the reaction. The obtained values are discussed in relation to the knowledge acquired from the literature. It should be mentioned that the study is limited to catalysts leading to a mixture of $E$ and $Z$ olefins due to thermodynamic equilibrium. The performances of $Z / E$ selective catalysts cannot be described by the kinetic parameters established here and are out of the scope of this study. 

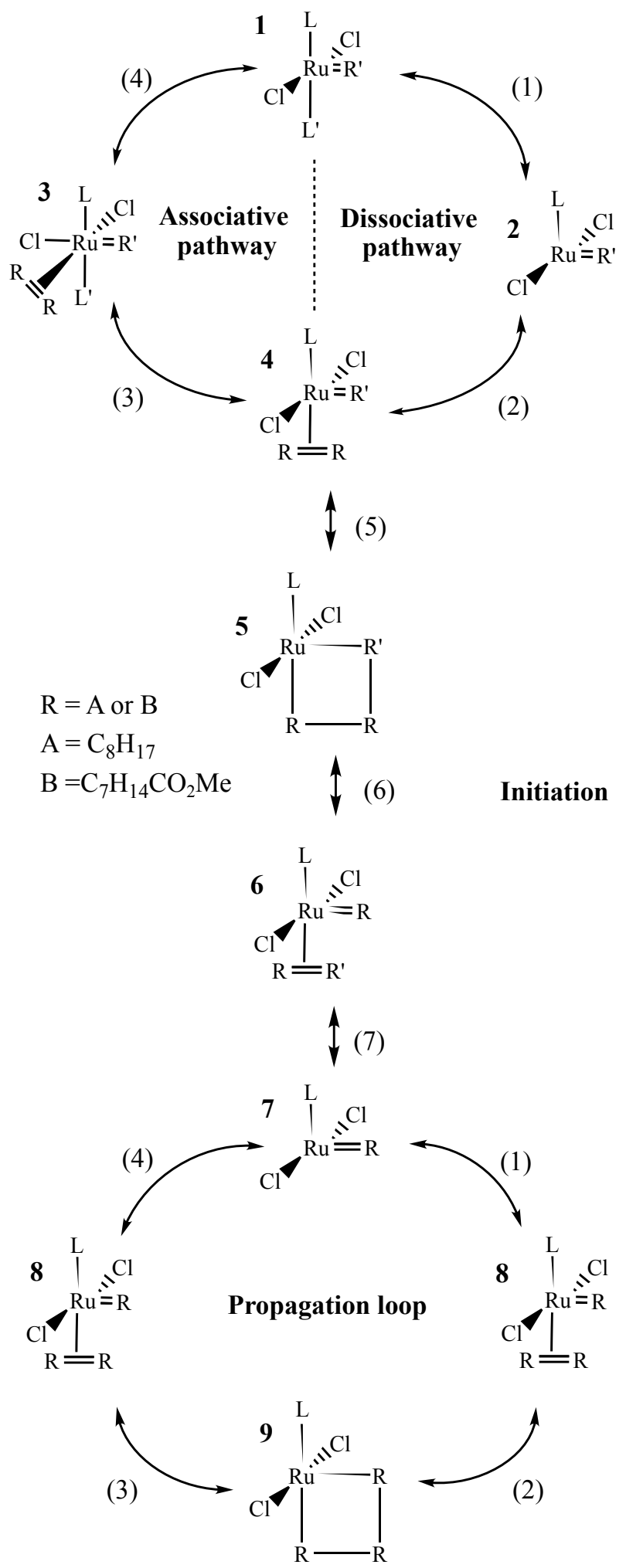

Scheme 2. Different elementary steps involved in the mechanism of the homo-metathesis reaction.

\section{Results}

The list of the used Ru-NHC pre-catalysts is presented in Figure 1. They have all the same $\mathrm{LL}^{\prime} \mathrm{Cl}_{2} \mathrm{Ru}=\mathrm{R}^{\prime}(\mathbf{1})$ general formula. They were selected to vary either the nature of the leaving ligand (L') or the bulkiness of the ancillary ligand (L). Thanks to the mechanism proposed in Scheme 2, 96 reaction steps were considered with 96 associated kinetic constants of which 24 steps for initiation, 24 steps 
for regeneration, and 48 steps for propagation (see the Supplementary Materials). The reaction rate of each step is given according to the mass action law. it is described by a first-order rate equation for both the catalyst and the olefin. In this kinetic model, it is presumed that there is no double bond migration (i.e., no olefin isomerization) and thus no secondary metathesis reactions. This consideration is supported by our experiments, which did not exhibit side products arising from these latter reactions (see a representative GC chromatogram in the Supplementary Materials).

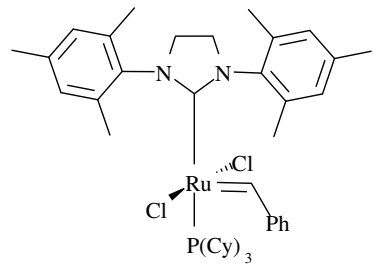

(G-II)

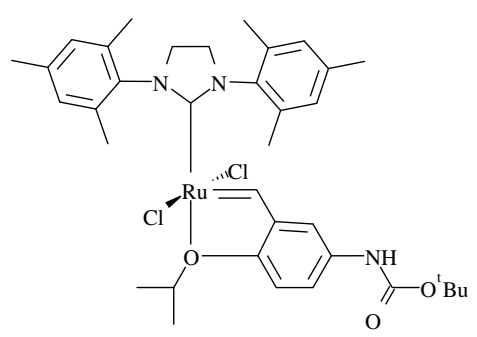

(M73-SIMes)

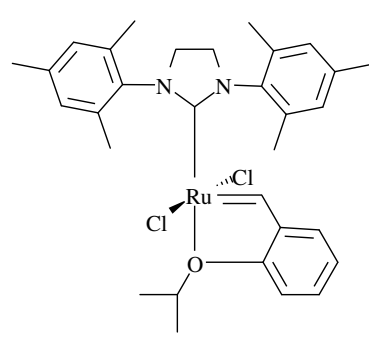

(HG-II)

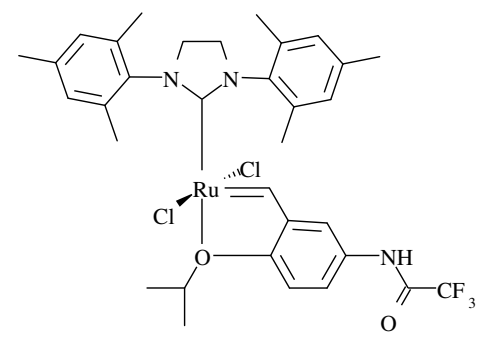

(M71-SIMes)

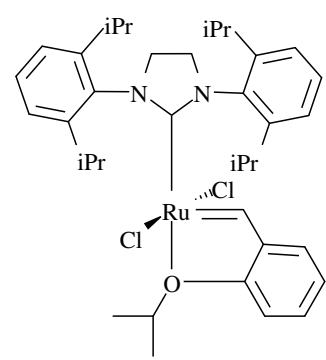

(HG-SIPr)

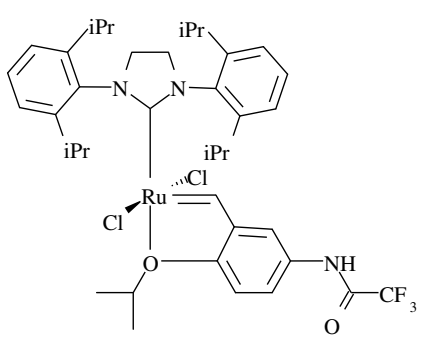

(M71-SIPr)

Figure 1. Ruthenium precatalysts.

One example of transformation is presented in Figure 2 for one catalyst (HG-II) using pure Z-olefin (methyl oleate) or pure E-olefin (methyl elaidate). In both cases, the same conversion and the same distribution of products are obtained at the end of reaction as defined by the thermodynamic equilibrium, indicating that the catalyst is not deactivated. Moreover, in both cases, an initiation period is noticeable, with an inflection point after several minutes. Another observation concerns the concentration of the symmetrical products; the concentrations of both $E$ products are equal $([\mathrm{AAE}]=[\mathrm{BBE}])$, as for the $Z$ products $([\mathrm{AAZ}]=[\mathrm{BBZ}])$ whatever the conversion. This last result contrasts with the results published by Kajetanowicz et al. [20] where side products are formed due to isomerization of the olefin. In our case, the formation of ruthenium hydride that were suspected to be responsible for the isomerization, is believed not to occur. The above observations are valid for all the pre-catalysts of Scheme 1.
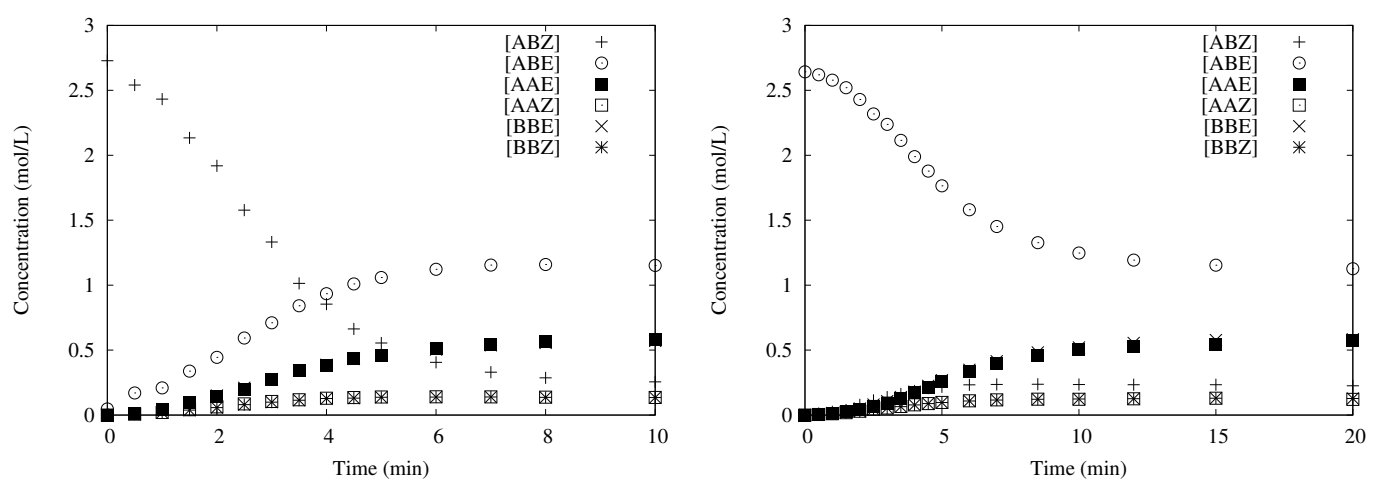

Figure 2. Transformation of methyl oleate with HG-II catalyst at $40^{\circ} \mathrm{C},[\mathrm{Ru}]=1.4 \times 10^{-5} \mathrm{~mol} / \mathrm{L}$, $[\mathrm{AB}]=2.7 \mathrm{~mol} / \mathrm{L}$ : (Left) starting from the Z-isomer; and (Right) starting from the $E$-isomer. 
The analysis of the literature prompted us to build a simplified kinetic model as outlined below:

(a) Ahr et al. [21] reported a very slight difference in the NMR chemical shifts of the $\mathrm{RuCl}_{2}(\mathrm{~L})=\mathrm{C}_{8} \mathrm{H}_{17}$ (noted RuA, A being the alkyl chain moiety) and $\mathrm{RuCl}_{2}(\mathrm{~L})=\mathrm{C}_{7} \mathrm{H}_{14} \mathrm{CO}_{2}$ Et (respectively, RuB, with $\mathrm{B}$ the ester moiety) alkylidene species (0.02 ppm); during the reaction, both species were also present in the same concentration. This strongly suggests that the Ru coordination sphere is not affected by the presence of an ester group (RuB) and that both species behave similarly. We can now write RuR to indifferently designate the alkylidene species RuA or RuB.

(b) The $E$ olefin configuration is thermodynamically favored. The $Z / E$ selectivity depends on the energy differences between the reaction pathways [22-24], and thus the kinetic constants of the reaction steps depend on the configuration of the reacting and the formed olefins. From a reactivity point of view and as fragments $\mathrm{A}$ and $\mathrm{B}$ are considered equivalent, all Z-olefins, i.e., $\mathrm{ABZ}, \mathrm{AAZ}$, and BBZ, are expected to have the same reactivity, thus a single kinetic constant is defined. A similar analysis stands for $\mathrm{ABE}, \mathrm{AAE}$, and $\mathrm{BBE}$.

(c) According to the metathesis mechanism shown in Scheme 2, the active species reacts with an olefin in the propagation loop. it could also react with $\mathrm{L}^{\prime}$ to regenerate the pre-catalyst. Depending on the ratio between the propagation rate and the regeneration rate, the pre-catalyst concentration after initiation can be either significant or negligible. For Grubbs second-generation catalysts, Sanford et al. [1] reported that the propagation and the regeneration kinetic constants are of the same order of magnitude. Then, at low catalyst loading in the reaction medium, and thus low concentration of initial ligand L' compared to the substrate, the propagation rate is high compared to the regeneration rate, and thus the regeneration step can be neglected (see Equation (1)).

$$
\frac{r_{\text {prop }}}{r_{\text {rege }}}=\frac{k_{\text {prop }}}{k_{\text {rege }}} \frac{[A B][R u R]}{\left[L^{\prime}\right][R u R]} \text {, with }\left[L^{\prime}\right]<<[A B]
$$

For Hoveyda second-generation catalysts, Bates et al. [25] studied the effect of adding some extra L' ligand to the reaction medium. For similar ligand and substrate concentrations, the propagation and regeneration rates were found similar. The conclusions given on the boomerang effect was for challenging substrates, were you have to work at high loading of catalyst. Here, we have very reactive substrates and work at a S/C ratio of 500,000. Thus, the assumption of irreversibility is completely valid. We can now consider the lumped mechanism derived from Scheme 2, presented in Scheme 3.

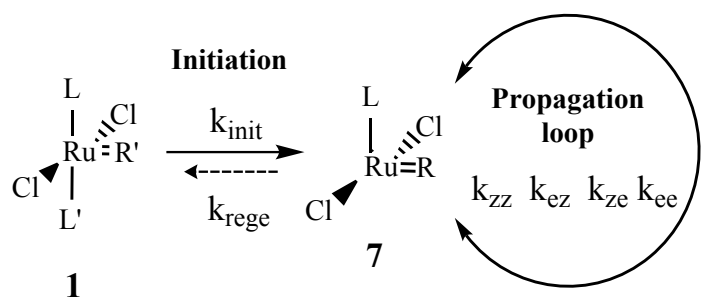

Scheme 3. Lumped catalytic cycle.

As a summary, the following hypotheses are considered:

- There is only one catalytic species, named RuR.

- $\quad$ All Z molecules have the same reactivity, as for all $E$ molecules (as shown in Scheme 4).

- The initiation reaction leading to the active species $\mathrm{RuR}$ is not reversible.

- No deactivation occurs. 


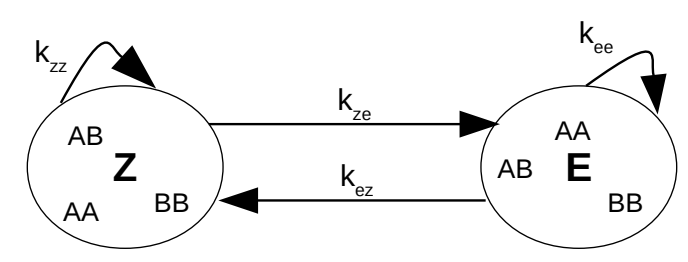

Scheme 4. Kinetic parameters for metathesis propagation reactions.

From this point, an enumeration of all possible reactions (productive metathesis, degenerated metathesis, and isomerization) was carried out. The complete list is provided in the Supplementary Materials.

The resulting mass balance system in the batch reactor used for the experiments can be expressed by the scalar product between a kinetic constant vector and a concentration vector:

$$
\frac{d C}{d t}=\vec{P} \cdot \vec{C} \cdot[R u R]
$$

resulting in the six following equations:

$$
\begin{aligned}
& \frac{d[A B Z]}{d t}=2\left(\begin{array}{c}
-k_{z z}-2 k_{z e} \\
k_{e z} \\
k_{z z} \\
k_{e z} \\
k_{z z} \\
k_{e z}
\end{array}\right)\left(\begin{array}{l}
{[A B Z]} \\
{[A B E]} \\
{[A A Z]} \\
{[A A E]} \\
{[B B Z]} \\
{[B B E]}
\end{array}\right)[R u R] \\
& \frac{d[A B E]}{d t}=2\left(\begin{array}{c}
k_{z e} \\
-k_{e e}-2 k_{e z} \\
k_{z e} \\
k_{e e} \\
k_{z e} \\
k_{e e}
\end{array}\right)\left(\begin{array}{l}
{[A B Z]} \\
{[A B E]} \\
{[A A Z]} \\
{[A A E]} \\
{[B B Z]} \\
{[B B E]}
\end{array}\right)[R u R] \\
& \frac{d[A A Z]}{d t}=\left(\begin{array}{c}
k_{z z} \\
k_{e z} \\
-2 k_{z z}-4 k_{z e} \\
2 k_{e z} \\
0 \\
0
\end{array}\right)\left(\begin{array}{l}
{[A B Z]} \\
{[A B E]} \\
{[A A Z]} \\
{[A A E]} \\
{[B B Z]} \\
{[B B E]}
\end{array}\right)[R u R] \\
& \frac{d[A A E]}{d t}=\left(\begin{array}{c}
k_{z e} \\
k_{e e} \\
2 k_{z e} \\
-2 k_{e e}-4 k_{e z} \\
0 \\
0
\end{array}\right)\left(\begin{array}{c}
{[A B Z]} \\
{[A B E]} \\
{[A A Z]} \\
{[A A E]} \\
{[B B Z]} \\
{[B B E]}
\end{array}\right)[R u R] \\
& \frac{d[B B Z]}{d t}=\left(\begin{array}{c}
k_{z z} \\
k_{e z} \\
0 \\
0 \\
-2 k_{z z}-4 k_{z e} \\
2 k_{e z}
\end{array}\right)\left(\begin{array}{c}
{[A B Z]} \\
{[A B E]} \\
{[A A Z]} \\
{[A A E]} \\
{[B B Z]} \\
{[B B E]}
\end{array}\right)[R u R]
\end{aligned}
$$




$$
\frac{d[B B E]}{d t}=\left(\begin{array}{c}
k_{z e} \\
k_{e e} \\
0 \\
0 \\
2 k_{z e} \\
-2 k_{e e}-4 k_{e z}
\end{array}\right)\left(\begin{array}{l}
{[A B Z]} \\
{[A B E]} \\
{[A A Z]} \\
{[A A E]} \\
{[B B Z]} \\
{[B B E]}
\end{array}\right)[R u R]
$$

Moreover,

$$
\frac{d[R u R]}{d t}=k_{\text {init }}\left(\left[L L^{\prime} C l_{2} R u R^{\prime}\right]_{0}-[R u R]\right)
$$

For pre-catalysts G-II, HG-II, M71-SIMes, and M73-SIMes, a single active species was considered (Figure 3) and, thus, identical propagation constants were considered for all those pre-catalysts albeit with different initiation constants. Using a set of experiments with pure ABE or pure ABZ olefins and different pre-catalysts, the resolution of the system of Equations (3)-(9) leading to four propagation constants $\left(k_{z z}, k_{z e}, k_{e e}\right.$, and $\left.k_{e z}\right)$ and four initiation constants (one per pre-catalyst) was implemented in the MATLAB R2016a release.

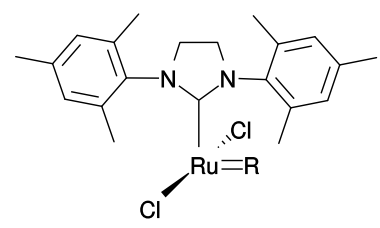

Figure 3. Active species formed from G-II, HG-II, M71-SIMes and M73-SIMes pre-catalysts. R stands for A or B fragments.

The kinetic model is a system of ordinary differential equations and is solved using an ode integration routine. The objective is to minimize the residual sum of squares (the residual being the difference between an experimental value, and the fitted value provided by the model) and is defined by the objective function presented in Equation (10). Note that the concentrations have been normalized by their maximal value in order to have the same statistical weight for each product.

$$
\mathrm{OF}=\sum_{i=1}^{N} \sum_{j=1}^{k}\left(C_{i j, \exp }-C_{i j, m o d}\right)^{2}
$$

where $\mathrm{N}$ is the number of experiments, $\mathrm{k}$ is the number of experimental data points for each experiment, and $C_{\text {exp }}$ and $C_{\text {mod }}$ are experimental and modeled normalized concentrations, respectively. The obtained parameters are listed in Table 1. it appears first that the initiation constants decrease in

\begin{tabular}{|c|c|c|c|c|c|}
\hline Pre-Catalyst & $\begin{array}{l}k_{\text {init }} \\
\left(\mathrm{s}^{-1}\right)\end{array}$ & $\begin{array}{c}k_{z z} \\
\left(\mathrm{~L} \cdot \mathrm{mol}^{-1} \cdot \mathrm{s}^{-1}\right)\end{array}$ & $\begin{array}{c}k_{z e} \\
\left(\mathrm{~L} \cdot \mathrm{mol}^{-1} \cdot \mathrm{s}^{-1}\right)\end{array}$ & $\begin{array}{c}k_{e z} \\
\left(\mathrm{~L} \cdot \mathrm{mol}^{-1} \cdot \mathrm{s}^{-1}\right)\end{array}$ & $\begin{array}{c}k_{e e} \\
\left(\mathrm{~L} \cdot \mathrm{mol}^{-1} \cdot \mathrm{s}^{-1}\right)\end{array}$ \\
\hline G-II & $1.8 \times 10^{-4} \pm 0.1 \times 10^{-4}$ & \multirow{4}{*}{$120 \pm 9$} & \multirow{4}{*}{$260 \pm 15$} & \multirow{4}{*}{$58 \pm 4$} & \multirow{4}{*}{$79 \pm 5$} \\
\hline HG-II & $3.2 \times 10^{-3} \pm 0.2 \times 10^{-3}$ & & & & \\
\hline M71-SIMes & $1.2 \times 10^{-2} \pm 0.1 \times 10^{-2}$ & & & & \\
\hline M73-SIMes & $1.9 \times 10^{-2} \pm 0.3 \times 10^{-2}$ & & & & \\
\hline
\end{tabular}
the following order: M73-SIMes > M71-SIMes > HG-II > G-II.

Table 1. Kinetic parameters obtained in the case of mesityl-substituted NHC ligands at $40^{\circ} \mathrm{C}$.

M73-SIMes initiates 100 times faster than G-II. Note that this result was obtained no matter the Z or $E$ configuration of the transformed olefin. In addition, the independence of the kinetic parameters has been checked as revealed in the correlation matrix and with the Student coefficient (see the Supplementary Materials). The value of $\mathrm{k}_{i n i}$ for the G-II catalyst $\left(1.8 \times 10^{-4} \mathrm{~s}^{-1}\right)$ is not too far from that determined by Grubbs $\left(4.6 \times 10^{-4} \mathrm{~s}^{-1}\right)$ considering the differences in the reaction conditions. 
Such a low value is attributed to the strong coordination of the basic $\mathrm{PCy}_{3}$ ligand [1]. All other catalysts bearing isopropoxybenzylidene ligands show much higher initiation constants, likely because of the facility for the isopropoxy group to decoordinate. There is however no clear quantitative relationship between $k_{i n i}$ and the $\sigma_{p}$ value of the para-substituting group $\mathrm{H}, \mathrm{NHCOCF}_{3}$ and $\mathrm{NHCO}_{2} \mathrm{tBu}$ in catalysts HG-II, M71-SIMes, and M73-SIMes, respectively. Concerning the propagation constants, they range as follows: $k_{z e}>k_{z z}>k_{e e}>k_{e z}$. Z-olefins are more easily transformed than their $E$-stereoisomer. This ranking and the relative values of the parameters are related to the thermodynamic equilibrium:

$$
\frac{[A B E]_{e q}}{[A B Z]_{e q}}=\frac{k_{z e}}{k_{e z}}
$$

From the optimized parameters, the equilibrium constant, $k_{z e} / k_{e z}=4.5 \pm 0.5$, is in total accordance with the experiments, where the equilibrium concentration ratio is $4.6 \pm 0.2$. This result, showing that all the pre-catalysts lead to the same equilibrium, is only valid for pre-catalysts showing no steric interactions on the Ru center (non-Z-selective catalysts). Note that obtaining kinetic selectivity through stereoselective retention [26] cannot be described using the hypotheses of the present paper.

Figure 4 presents the parity diagram between the experimental normalized concentrations and the modeled ones using the parameters given in Table 1. The ca. 700 points correspond to 4 pre-catalysts, 2 series of experiments per pre-catalyst (starting from pure ABE and pure ABZ), and 14 samples per experiment with 6 molecules per sample. A very good fit is obtained between the experimental concentrations and the calculated ones with values within an error of $10 \%$.

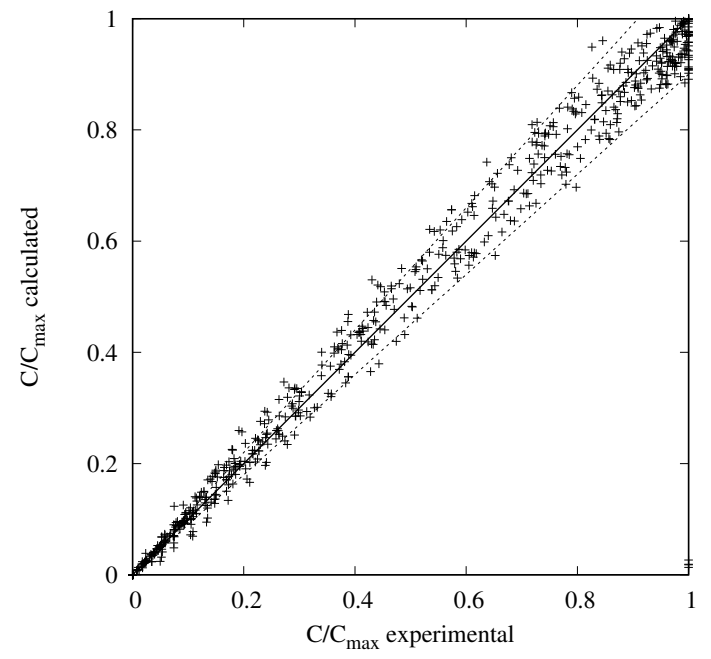

Figure 4. Parity plot between modeled and experimental normalized concentrations $( \pm 10 \%)$.

Thus, the strong hypothesis that the propagation constants are the same for all catalysts and for $\mathrm{ABE}$ or $\mathrm{ABZ}$ reactants is not called into question. These results reinforce our hypotheses and allow concluding that the huge difference of reactivity between the $Z$ and the $E$ molecule can only be due to strong differences in the kinetic parameters. The ranking of the initiation constants, varying in a range of two orders of magnitude, is explained by the easier decoordination of the pincer ether than the phosphine group, and, further, by the presence of attracting groups on the benzylidene unit. A value of $2.4 \times 10^{-3} \mathrm{~min}^{-1}$ (i.e., $4 \times 10^{-5} \mathrm{~s}^{-1}$ ) was published for the initiation constant of HG-II $[14,18]$ at $10^{\circ} \mathrm{C}$, a value 80 times lower than that obtained here at $40^{\circ} \mathrm{C}$. This apparent discrepancy is related to operating conditions (especially the temperature but also the concentrations and species in solution, among others) that differ in both studies.

The same modeling work was performed from experiments performed with pre-catalysts bearing di-isopropyl-phenyl-substituted NHC groups, thus leading to another active species (see Figure 5). In this new set of experiments, four other propagation constants were estimated and two initiation 
constants for the two pre-catalysts. The results are presented in Table 2. The initiation constants show the same ranking as in Table 1: the complex bearing a trifluoroamido-substituted styrenylether initiates ca. 4 times faster than the non-substituted one in both cases. Comparing now the values of the initiation constants of two pre-catalysts bearing the same styrenylether group but different NHC substituents (e.g., HG-II and HG-SIPr), the hindered NHC pre-catalyst was found to initiate much more slowly than the less hindered one. One order of magnitude difference is observed between the initiation constants of both pre-catalysts, in complete accordance with what was observed by Grubbs et al. [14,18]. The propagation constants were in the same order of magnitude as those observed with the mesityl-substituted NHC-Ru pre-catalysts. As the number of experiments was insufficient to ensure that the parameters are determined independently (see correlation matrix in the Supplementary Materials), a further interpretation would be highly questionable.

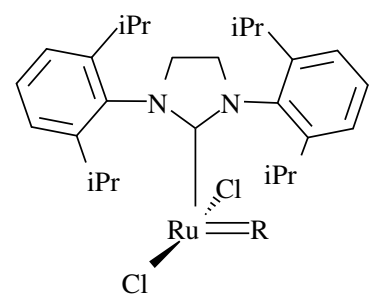

Figure 5. Active species formed from HG-SIPr and, M71-SIPr pre-catalysts. R stands for A or B fragments.

Table 2. Kinetic parameters obtained in the case of isopropyl-substituted NHC ligands at $40{ }^{\circ} \mathrm{C}$.

\begin{tabular}{|c|c|c|c|c|c|}
\hline Pre-Catalyst & $\begin{array}{l}k_{\text {init }} \\
\left(\mathrm{s}^{-1}\right)\end{array}$ & $\begin{array}{c}k_{z z} \\
\left(\mathrm{~L} \cdot \mathrm{mol}^{-1} \cdot \mathrm{s}^{-1}\right)\end{array}$ & $\begin{array}{c}k_{z e} \\
\left(\mathrm{~L} \cdot \mathrm{mol}^{-1} \cdot \mathrm{s}^{-1}\right)\end{array}$ & $\begin{array}{c}k_{e z} \\
\left(\mathrm{~L} \cdot \mathrm{mol}^{-1} \cdot \mathrm{s}^{-1}\right)\end{array}$ & $\begin{array}{c}k_{e e} \\
\left(\mathrm{~L} \cdot \mathrm{mol}^{-1} \cdot \mathrm{s}^{-1}\right)\end{array}$ \\
\hline $\begin{array}{l}\text { HG-SIPr } \\
\text { M71-SIPr }\end{array}$ & $\begin{array}{l}1.8 \times 10^{-4} \pm 0.9 \times 10^{-4} \\
7.3 \times 10^{-4} \pm 0.5 \times 10^{-4}\end{array}$ & $325 \pm 5$ & $650 \pm 10$ & $190 \pm 5$ & $410 \pm 15$ \\
\hline
\end{tabular}

The computed concentrations vs. time profiles could be compared to the experimental ones, as shown in Figure 6 in the case of M71-SIPr catalyst. Again, the transformation of ABZ and ABE was satisfactorily described with the same five kinetic parameters. The slowest reactivity of $A B E$ was not due to the initiation, which is exactly the same as for $\mathrm{ABZ}$, but to $k_{e z}$ which was weaker than $k_{z z}$.
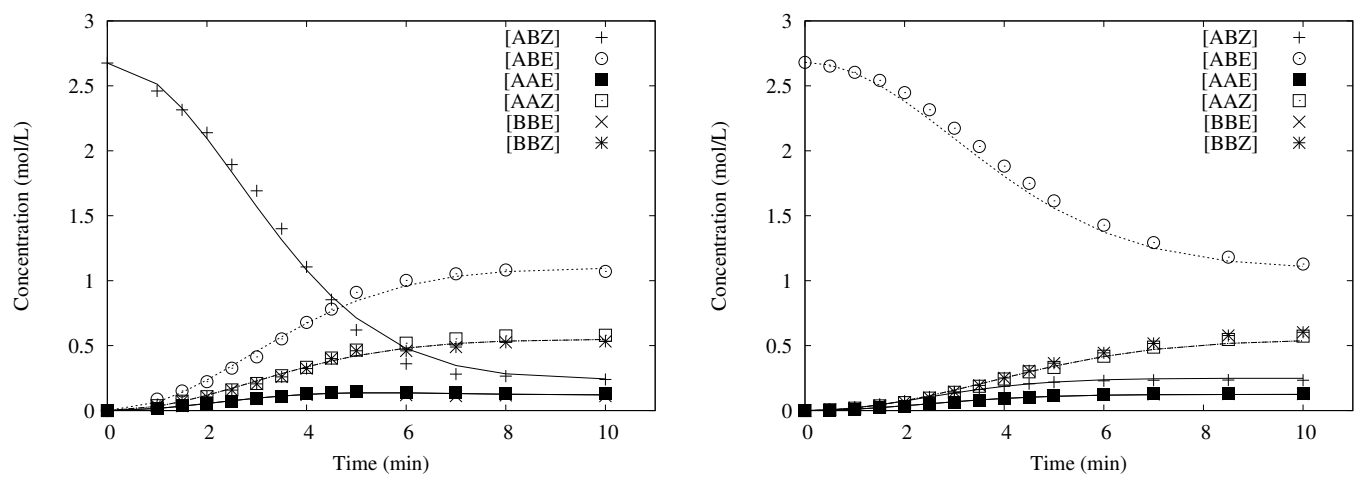

Figure 6. Computed and experimental concentration profiles for M71-SIPr pre-catalyst at $40^{\circ} \mathrm{C}$, $[\mathrm{Ru}]=1.4 \times 10^{-5} \mathrm{~mol} / \mathrm{L},[\mathrm{AB}]=2.7 \mathrm{~mol} / \mathrm{L}$ : (Left) starting from the Z-isomer; and (Right) starting from the $E$-isomer. Lines represent the modeled concentrations (discrete values connected with straight lines).

In this context, faster initiation could be obtained by replacing the isopropyl group bonded to the oxygen by selected saturated or unsaturated derivatives of cyclopropane, cyclobutane, 
and cyclopentane [17]. The authors showed that introducing a double bond into the cycloalkyl group weakens the ruthenium-oxygen bond, creating a series of potential catalysts capable of fast initiation metathesis.

\section{Materials and Methods}

All catalytic tests were carried out inside a glovebox filled with argon. Circa $1.50 \mathrm{~g}$ of substrate and $150 \mathrm{mg}$ of methyl heptadecanoate (internal standard) were weighed inside a $5 \mathrm{~mL}$ vial. The vial was capped with a septum and the mixture was stirred in a sand bath at $40^{\circ} \mathrm{C}$ for $20 \mathrm{~min}$. Then, $20 \mu \mathrm{L}$ of a stock solution of the catalyst in dichloromethane (the concentration was adjusted depending on the desired substrate/catalyst ratio) was added and the reaction mixture was stirred at $40^{\circ} \mathrm{C}$ and $1000 \mathrm{rpm}$. The metathesis reaction was monitored by sampling through the septum at suitable intervals, and each aliquot was immediately quenched by an excess of ethyl vinyl ether placed in a capped GC vial. Samples were analyzed by GC on an Agilent 7890A equipped with a FID detector and DB23 column $(50 \mathrm{~m} \times 0.25 \mathrm{~mm})$. Oven program: $80^{\circ} \mathrm{C}(5 \mathrm{~min}), 10^{\circ} \mathrm{C} / \mathrm{min}, 150^{\circ} \mathrm{C}(5 \mathrm{~min}), 10^{\circ} \mathrm{C} / \mathrm{min}$, and $250^{\circ} \mathrm{C}(11 \mathrm{~min})$. Retention times ( $\mathrm{min}): 18.2$ (E-9-octadecene), 18.3 (Z-9-octadecene), 25.3 (methyl heptadecanoate), 26.4 (methyl elaidate), 26.5 (methyl oleate), 33.3 (dimethyl E-9-octadecene-1,18-dioate), and 33.5 (dimethyl Z-9-octadecene-1,18-dioate). A representative gas chromatogram is provided in the Supplementary Materials.

\section{Conclusions}

This work demonstrates that, for the metathesis of internal olefins, a simple kinetic model considering three reasonable hypotheses, one initiation constant, and four propagation constants could account for a large set of up to 700 experimental data involving six pre-catalysts. In the case of non-stereoretentive catalysts (no steric hindrance on the $\mathrm{Ru}$ ), the nature of the leaving alkylidene ligand drives the initiation rate only, whereas the NHC group has an influence on both the initiation and propagation rates. Whatever the pre-catalyst, the ranking between the propagation constants is the same. The transformation rates of $Z$ and $E$ isomers is only driven by these propagation constants and nothing differentiates the initiation step. Considering that the active species is the same for all pre-catalysts with only a variation of $\mathrm{L}^{\prime}$, using immobilized catalysts by anchoring through the NHC ligand would lead to the same performances after initiation. On the contrary, in a batch reactor, or in a reactor with a circulation of the catalyst, a high initiation rate is beneficial. Furthermore, the leaving initial alkylidene moiety would be washed out of the reactor, preventing any backward reaction. Future works will be devoted to broaden the scope of this model to other catalysts, including fast-initiating pyridine Grubbs third-generation catalysts [27] and olefins.

Supplementary Materials: The following are available at http:/ / www.mdpi.com/2073-4344/10/4/435/s1

Author Contributions: Conceptualization, C.d.B. and C.T.; methodology, C.d.B. and C. T.; software, M.H. and C.N.; formal analysis, C.N., M.H., and V.M.; investigation, M.R.C. and M.H.; writing-original draft preparation, V.M., M.H., and M.R.C.; writing—review and editing, V.M.; visualization, V.M., C.N., and M.H.; supervision, V.M., C.T., and C.d.B.; project administration, V.M. and C.T.; and funding acquisition, V.M. and C.T. All authors have read and agreed to the published version of the manuscript.

Funding: Part of this research was funded by the French "Agence Nationale de la Recherche" in the framework of the project ANR-12-CDII-0002 CFLOW-OM.

Acknowledgments: Umicore PMC (P. Oczipka) is acknowledged for the gift of M7 pre-catalysts. BASF (P. Hervy) is acknowledged for the gift of the Selexsorb® CD.

Conflicts of Interest: The authors declare no conflict of interest. 


\section{References}

1. Sanford, M.S.; Love, J.A.; Grubbs, R.H. Mechanism and Activity of Ruthenium Olefin Metathesis Catalysts. J. Am. Chem. Soc. 2001, 123, 6543-6554, doi:10.1021/ja010624k. [CrossRef] [PubMed]

2. Van der Gryp, P.; Marx, S.; Vosloo, H.C.M. Experimental, DFT and Kinetic Study of 1-Octene Metathesis with Hoveyda-Grubbs Second Generation Precatalyst. J. Mol. Catal. Chem. 2012, 355, 85-95, doi:10.1016/j.molcata.2011.12.001. [CrossRef]

3. du Toit, J.I.; van der Gryp, P.; Loock, M.M.; Tole, T.T.; Marx, S.; Jordaan, J.H.L.; Vosloo, H.C.M. Industrial Viability of Homogeneous Olefin Metathesis: Beneficiation of Linear Alpha Olefins with the Diphenyl-Substituted Pyridinyl Alcoholato Ruthenium Carbene Precatalyst. Catal. Today 2016, 275, 191-200, doi:10.1016/j.cattod.2015.12.004. [CrossRef]

4. Chikkali, S.; Mecking, S. Refining of Plant Oils to Chemicals by Olefin Metathesis. Angew. Chem. Int. Ed. 2012, 51, 5802-5808, doi:10.1002/anie.201107645. [CrossRef] [PubMed]

5. Scott, S. Catalytic transformation of seed oil derivatives via olefin metathesis. HELIA 2014, 30, 133-142, doi:10.2298/hel0746133s. [CrossRef]

6. Higman, C.S.; Lummiss, J.A.M.; Fogg, D.E. Olefin Metathesis at the Dawn of Implementation in Pharmaceutical and Specialty-Chemicals Manufacturing. Angew. Chem. Int. Ed. 2016, 55, 3552-3565, doi:10.1002/anie.201506846. [CrossRef]

7. Dinger, M.; Mol, J. High Turnover Numbers with Ruthenium-Based Metathesis Catalysts. Adv. Synth. Catal. 2002, 344, 671-677, doi:10.1002/1615-4169(200208)344:6/7<671::AID-ADSC671>3.0.CO;2-G. [CrossRef]

8. Marvey, B.B.; Segakweng, C.K.; Vosloo, M.H.C. Ruthenium Carbene Mediated Metathesis of Oleate-Type Fatty Compounds. Int. J. Mol. Sci. 2008, 9, 615-625, doi:10.3390/ijms9040615. [CrossRef]

9. Thomas, P.A.; Marvey, B.B. Room Temperature Ionic Liquids as Green Solvent Alternatives in the Metathesis of Oleochemical Feedstocks. Molecules 2016, 21, 184, doi:10.3390/molecules21020184. [CrossRef]

10. Zelin, J.; Trasarti, A.F.; Apesteguía, C.R. Self-metathesis of methyl oleate on silica-supported Hoveyda-Grubbs catalysts. Catal. Commun. 2013, 42, 84-88, doi:10.1016/j.catcom.2013.08.007. [CrossRef]

11. Pillai, S.K.; Hamoudi, S.; Belkacemi, K. Functionalized Value-Added Products via Metathesis of Methyloleate over Methyltrioxorhenium Supported on ZnCl2-Promoted Mesoporous Alumina. Fuel 2013, 110, 32-39, doi:10.1016/j.fuel.2012.10.040. [CrossRef]

12. Salameh, A.; Baudouin, A.; Basset, J.M.; Copéret, C. Tuning the Selectivity of Alumina-Supported (CH3)ReO3 by Modifying the Surface Properties of the Support. Angew. Chem. Int. Ed. 2008, 47, 2117-2120, doi:10.1002/anie.200704876. [CrossRef] [PubMed]

13. Hasib-ur Rahman, M.; Hamoudi, S.; Belkacemi, K. Fatty Acid Methyl Ester Heterogeneous Self-metathesis in Hydrophobic Green Solvent: Mass Transfer Limitations, Catalyst Recyclability, and Stability. Can. J. Chem. Eng. 2018, 96, 223-230, doi:10.1002/cjce.22918. [CrossRef]

14. Engle, K.M.; Lu, G.; Luo, S.X.; Henling, L.M.; Takase, M.K.; Liu, P.; Houk, K.N.; Grubbs, R.H. Origins of Initiation Rate Differences in Ruthenium Olefin Metathesis Catalysts Containing Chelating Benzylidenes. J. Am. Chem. Soc. 2015, 137, 5782-5792, doi:10.1021/jacs.5b01144. [CrossRef] [PubMed]

15. Thiel, V.; Hendann, M.; Wannowius, K.J.; Plenio, H. On the Mechanism of the Initiation Reaction in Grubbs-Hoveyda Complexes. J. Am. Chem. Soc. 2012, 134, 1104-1114, doi:10.1021/ja208967h. [CrossRef]

16. Liu, P.; Xu, X.; Dong, X.; Keitz, B.K.; Herbert, M.B.; Grubbs, R.H.; Houk, K.N. Z-Selectivity in Olefin Metathesis with Chelated Ru Catalysts: Computational Studies of Mechanism and Selectivity. J. Am. Chem. Soc. 2012, 134, 1464-1467, doi:10.1021/ja2108728. [CrossRef]

17. Trzaskowski, B.; Goddard, W.A.; Grela, K. Faster Initiating Olefin Metathesis Catalysts from Introducing Double Bonds into Cyclopropyl, Cyclobutyl and Cyclopentyl Derivatives of Hoveyda-Grubbs Precatalysts. Mol. Catal. 2017, 433, 313-320, doi:10.1016/j.mcat.2016.12.018. [CrossRef]

18. Luo, S.X.; Engle, K.M.; Dong, X.; Hejl, A.; Takase, M.K.; Henling, L.M.; Liu, P.; Houk, K.N.; Grubbs, R.H. An Initiation Kinetics Prediction Model Enables Rational Design of Ruthenium Olefin Metathesis Catalysts Bearing Modified Chelating Benzylidenes. ACS Catal. 2018, 8, 4600-4611, doi:10.1021/acscatal.8b00843. [CrossRef]

19. Benitez, D.; Tkatchouk, E.; Goddard, W.A. III. Relevance of cis- and trans-dichloride Ru intermediates in Grubbs-II olefin metathesis catalysis (H2IMesCl2RuCHR). Chem. Commun. 2008, 6194-6196, doi:10.1039/B815665D. [CrossRef] 
20. Kajetanowicz, A.; Sytniczuk, A.; Grela, K. Metathesis of renewable raw materials-Influence of ligands in the indenylidene type catalysts on self-metathesis of methyl oleate and cross-metathesis of methyl oleate with (Z)-2-butene-1,4-diol diacetate. Green Chem. 2014, 16, 1579-1585, doi:10.1039/C3GC42194E. [CrossRef]

21. Ahr, M.; Thieuleux, C.; Copéret, C.; Fenet, B.; Basset, J.M. Noels' vs. Grubbs' Catalysts: Evidence for One Unique Active Species for Two Different Systems! Adv. Synth. Catal. 2007, 349, 1587-1591, doi:10.1002/adsc.200700149. [CrossRef]

22. Dang, Y.; Wang, Z.X.; Wang, X. A Thorough DFT Study of the Mechanism of Homodimerization of Terminal Olefins through Metathesis with a Chelated Ruthenium Catalyst: From Initiation to Z Selectivity to Regeneration. Organometallics 2012, 31, 7222-7234, doi:10.1021/om300784k. [CrossRef]

23. Keitz, B.K.; Endo, K.; Patel, P.R.; Herbert, M.B.; Grubbs, R.H. Improved Ruthenium Catalysts for Z-Selective Olefin Metathesis. J. Am. Chem. Soc. 2012, 134, 693-699, doi:10.1021/ja210225e. [CrossRef] [PubMed]

24. Luján, C.; Nolan, S.P. E/Z selectivity in ruthenium-mediated cross metathesis. Catal. Sci. Technol. 2012, 2, 1027-1032, doi:10.1039/C2CY00457G. [CrossRef]

25. Bates, J.M.; Lummiss, J.A.M.; Bailey, G.A.; Fogg, D.E. Operation of the Boomerang Mechanism in Olefin Metathesis Reactions Promoted by the Second-Generation Hoveyda Catalyst. ACS Catal. 2014, 4, 2387-2394, doi:10.1021/cs500539m. [CrossRef]

26. Johns, A.M.; Ahmed, T.S.; Jackson, B.W.; Grubbs, R.H.; Pederson, R.L. High Trans Kinetic Selectivity in Ruthenium-Based Olefin Cross-Metathesis through Stereoretention. Org. Lett. 2016, 18, 772-775, doi:10.1021/acs.orglett.6b00031. [CrossRef]

27. Forcina, V.; García-Domínguez, A.; Lloyd-Jones, G.C. Kinetics of Initiation of the Third Generation Grubbs Metathesis Catalyst: Convergent Associative and Dissociative Pathways. Faraday Discuss. 2019, 220, 179-195, doi:10.1039/C9FD00043G. [CrossRef]

(C) 2020 by the authors. Licensee MDPI, Basel, Switzerland. This article is an open access article distributed under the terms and conditions of the Creative Commons Attribution (CC BY) license (http://creativecommons.org/licenses/by/4.0/). 